\title{
Multiplicity of nuclear dust lanes and dust lane shocks in the Milky Way bar
}

\author{
H. S. Liszt \\ National Radio Astronomy Observatory, 520 Edgemont Road, Charlottesville, VA 22903-2475, USA \\ e-mail: hliszt@nrao.edu \\ Received 8 March 2008 / Accepted 18 May 2008

\section{ABSTRACT}

\begin{abstract}
Aims. We show the existence of a small family of inner-galaxy dust lanes and dust lane standing shocks beyond the two major ones that were previously known to exist

Methods. We analyze images of CO emission in the inner regions of the Galaxy

Results. The peculiar kinematics of the major dust lane features are repeated in several other distinct instances at $l>0^{\circ}$, in one case at a contrary location $100 \mathrm{pc}$ above the galactic equator at $l>3^{\circ}$ at the upper extremity of Clump 2. Like the previously-known dust lanes, these new examples are also associated with localized, exceptionally broad line profiles believed to be characteristic of the shredding of neutral gas at the standing dust lane shocks.

Conclusions. There may be secondary dust lane and standing shocks in the Milky Way bulge. The vertical structure provides a temporal sequence for understanding the secular evolution of gas flow in the bar.
\end{abstract}

Key words. Galaxy: center - Galaxy: kinematics and dynamics

\section{Introduction}

Constructing a coherent picture of the inner-galaxy gas and its kinematics from our vantage point within the disk has proved challenging. In the earliest days the gas was first observed in $\mathrm{H} \mathrm{I}$ and decomposed into "features" (van der Kruit 1970; Cohen \& Davies 1976; Oort 1977) then subsequently observed in molecular lines (Fig. 1) revealing somewhat different, often seemingly disparate behavior. The various views were compared and reconciled (Burton \& Liszt 1983), largely through realization of the differences in the conditions of observing of various gas constituents, and the "features" were associated with each other and then with an underlying physical entity, namely a galactic bar. The process of observing, comparing and assimilating other constituents of the bar is ongoing, for instance with derivation of the distribution of extinction and dust from the bar dust lanes (Marshall et al. 2008) and study of the chemistry based on a dynamical separation of consituents (Rodriguez-Fernandez et al. 2006).

Along the way there were many false starts but the notion (which took hold very early) that the observations must be reconciled with the existence of a galactic bar in the presence of an overall tilt of the gas layer (Cohen \& Davies 1976; Burton \& Liszt 1978) certainly proved correct. Figure 1, a scan along the inner galactic plane in $\mathrm{CO}$, integrated over $-1^{\circ} \leq b \leq 1^{\circ}$ to suppress the wealth of vertical structure, illustrates the complexities which must be overcome in order to gain a view of the underlying physical phenomena. It also helps to explain why our understanding of the observations has improved as models of the bar gas flow have matured, from simple recognition of the $x_{2}-x_{1}$ orbit separation in fast bars (Binney et al. 1991) to holistic views of the hydrodynamic gas flow over an entire bar (Fux 1999; Regan et al. 1999; Regan \& Teuben 2003). For recent views of the galactic center gas distribution which incorporate

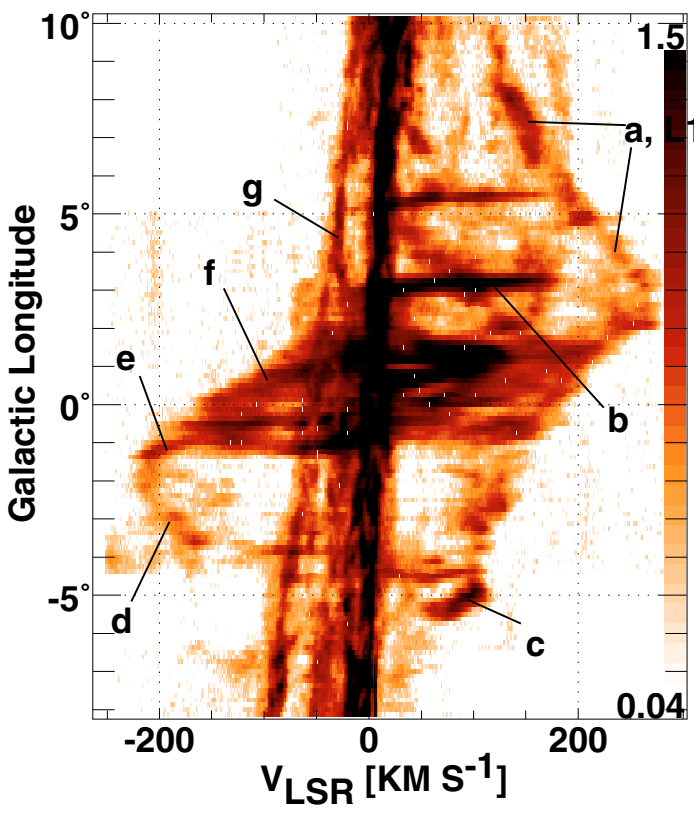

Fig. 1. Longitude-velocity diagram of ${ }^{12} \mathrm{CO}$ emission from the dataset of Bitran et al. (1997) on a $0.125^{\circ}$ grid, separately averaged over $-1^{\circ} \leq b \leq 1^{\circ}$ at each longitude. Features a (aka the "connecting arm") and $\mathrm{d}$ are associated with prominent galactic dust lanes; $\mathrm{b}$ and $\mathrm{c}$ are Clump 2 and Clump 1 of Bania (1977), e is the "rotating nuclear disk" of Rougour and Oort (Oort 1977), f is the "expanding molecular ring" of Kaifu et al. (1972) and g arises in the 3-kpc arm Bania (1980).

notions of bar gas flow, see Sawada et al. (2004); Liszt (2006); Rodriguez-Fernandez et al. (2006) and Marshall et al. (2008).

It is access to the rather extravagant vertical structure of the inner-galaxy gas which is the truly unique aspect of observing the galactic nucleus from the vantage point of the Sun, and this 
should feed back into models of the gas flow. However, the vertical structure has proved largely intractable theoretically and has often been suppressed (as in Fig. 1), ignored or denied, even in purely observational descriptions. Yet, it is the vertical structure of the gas which often presents the sharpest and most surprising structure (Liszt 2006). Marshall et al. (2008) demonstrated a vertical separation between the tilts of the dust and molecular gas in the Milky Way dust lane which would be important in interpreting observations of other systems.

Here we note that the peculiar kinematics which distinguish the standing shocks of the large-scale inner-galaxy bar dust lanes are repeated several times over the inner regions, in one case with a tilt quite opposite to that which is usually recognized. Apparently, the Milky Way has secondary bar/dust lane structures, as discussed in Sect. 2.

\section{Kinematic features associated with the dust lanes and dust lane shocks}

The dataset employed here is that of Bitran et al. (1997), with ${ }^{12} \mathrm{CO}$ spectra on a $0.125^{\circ}$ grid at $|b| \leq 1^{\circ},|l| \leq 13^{\circ}$ and on a $0.25^{\circ}$ grid at $1^{\circ} \leq|b| \leq 2^{\circ}$. To produce Fig. 1 we averaged (unweighted) over all latitudes at each longitude; this figure is similar to one panel of Fig. 3 of Marshall et al. (2008). Several of the most prominent kinematic features arising exterior to the Sgr A-E source complex are called out in Fig. 1.

\subsection{Kinematics of dust line features}

Despite their obvious asymmetries in intensity, velocity, and longitude extent, it is the paired features a and $d$ which traditionally have been associated with each other and with the near and far-side galactic dust lanes, respectively (Cohen \& Davies 1976; Liszt \& Burton 1980; Fux 1999; Rodriguez-Fernandez et al. 2006; Marshall et al. 2008). Features a and d are oppositely displaced below and above the galactic equator, respectively, in the sense of the most pervasive gas tilt (Liszt \& Burton 1980).

The a-feature was sufficiently prominent in $\mathrm{HI}$ to have been known originally as the "connecting arm" while the d-feature was not always remarked as a separate entity (see Fig. 1 of Cohen \& Davies 1976). It is a somewhat of an accident of the Sun's location that the dust lane shock appears in such a way as to form the terminal velocity. Incorporating the contrary kinematics of the a and d-features into the derivation of a galactic rotation curve directly from the observed terminal velocity, assuming pure circular motion, causes an apparent decline in equilibrium circular velocity with galactocentric radius, corrupting the mass model (Caldwell \& Ostriker 1981; Burton \& Liszt 1993).

Feature $f$ (the expanding molecular ring) and its positivevelocity counterpart crossing zero-longitude at $+165 \mathrm{~km} \mathrm{~s}^{-1}$ arise in the spray of material flowing inward along the dust lanes where they contact the nuclear star-forming ring containing the Sgr A-E radiocontinuum sources, see Regan et al. (1999), Fig. 9, or Fux (1999), Fig. 17. Sgr B2, which has been described as resulting from a cloud-cloud collision (Hasegawa et al. 2008), is probably at such a contact point.

Several other prominent features in Fig. 1 are directly related to the dust lanes, in particular the b-feature, Clump 2 of (Bania 1977; Stark \& Bania 1986), and the broad, localized, anonymous feature at $l=5.5^{\circ}$. These were mapped recently in several molecules at $1^{\prime}$ resolution (Liszt 2006) and discussed in terms of the shredding of large quantities of molecular gas at the standing dust lane shock (Fux 1999): various estimates yield some $10^{6} M_{\text {sun }}$ in either feature. The broad lines in Fig. 1 are spatially resolved at arcminute $(2.4 \mathrm{pc})$ resolution, with velocity gradients stronger than seen across the circumnuclear disk around SgrA*.

The weakness of the far-side dust lane feature $d$ at negative longitude may be related to the absence of similar broad-lined features at negative longitude, indicating that the negative longitude dust lane is (temporarily?) somewhat starved of gas.

\subsection{Other dust lane features}

Figure 2 shows the higher-velocity portions of two composite $l-v$ diagrams constructed below and above the galactic equator, at left and right, respectively. Like Fig. 1 these have also been integrated in latitude, but over smaller regions. At negative latitudes in the left-hand panel, a locus labelled L3, nearly parallel to the connecting arm feature, is prominent; it is of course faintly visible in Fig. 1 as well and was labelled feature $J$ by Rodriguez-Fernandez et al. (2006). It follows the most prominent gas tilt to negative latitude at $l>0$. Further mimicing the larger dust lane, there also appears to be a localized broad line over the interval down to 0 -velocity at $l=3^{\circ}$.

The span of L3 appears contained between the $l=5.5^{\circ}$ feature at one end $\left(l=5^{\circ}, v=0 \mathrm{~km} \mathrm{~s}^{-1}\right)$ and the strong-lined gas stretching back to $\operatorname{Sgr} \mathrm{A}$ at the other $\left(l=1.5^{\circ}, v=140 \mathrm{~km} \mathrm{~s}^{-1}\right)$. The region at $l=1.3^{\circ}$ is well-known for hosting a copious amount of gas and has been suggested as the contact point for inflowing material (Fux 1999). It was recently mapped by Tanaka et al. (2007) and attributed to a nascent superbubble, although there is no evidence for recent or current star formation activity. A broad-lined, vertically-extended dustlane standing shock feature forms the core of the gas concentration at this longitude (Liszt 2006).

At positive latitude $b=0.5^{\circ}-1.0^{\circ}$ in the right panel, Fig. $2 \mathrm{~b}$ is much leaner and dominated by several (3-4) of the localized broad-lined features. At $l>0^{\circ}$, all of the behavior in Fig. $2 \mathrm{~b}$ is spectacularly contrary to the overall general tilt of the gas layer. The locus L2, which is not readily apparent in Fig. 1, is clearly associated with the b-feature (Clump 2), in fact at its northern terminus. A broad-lined dust lane shock feature at $l=1.3^{\circ}$ is more prominent than in Fig. 2a because of its vertical extension. The gas at $l<-2^{\circ}$, including Clump 1 of Bania (1977), is a composite projection of a rear-side dust lane and the base of a far-side spiral arm, according to Fux (1999).

\subsection{Locating the dust lane features in space}

To locate the dust lane features, we created maps of the brightness integrated across the velocity ranges of their ridges in Fig. 2, varying the span of the integration with longitude (only). This is most nearly definitive for the connecting arm feature L1 because it occurs at the extremity of the gas kinematics. For the other dust lane features, gas associated with other aspects of the distribution is unavoidably captured, but this has the virtue that it exposes the relationships between the dust lanes and the shredded molecular gas.

Figure 3 has three panels corresponding to integration over the velocity spans of the L1-L3 features in Fig. 2. The L1 panel at top is similar to one panel of Fig. 3 of Marshall et al. (2008) because it represents the gas in the major dust lane. At its southernmost terminus at $l=5.5^{\circ}, b=-1.8^{\circ}$, the gas is $250 \mathrm{pc}$ below the galactic equator at a distance of $7.5 \mathrm{kpc}$ from the Sun (the dust lane points mostly toward us). 


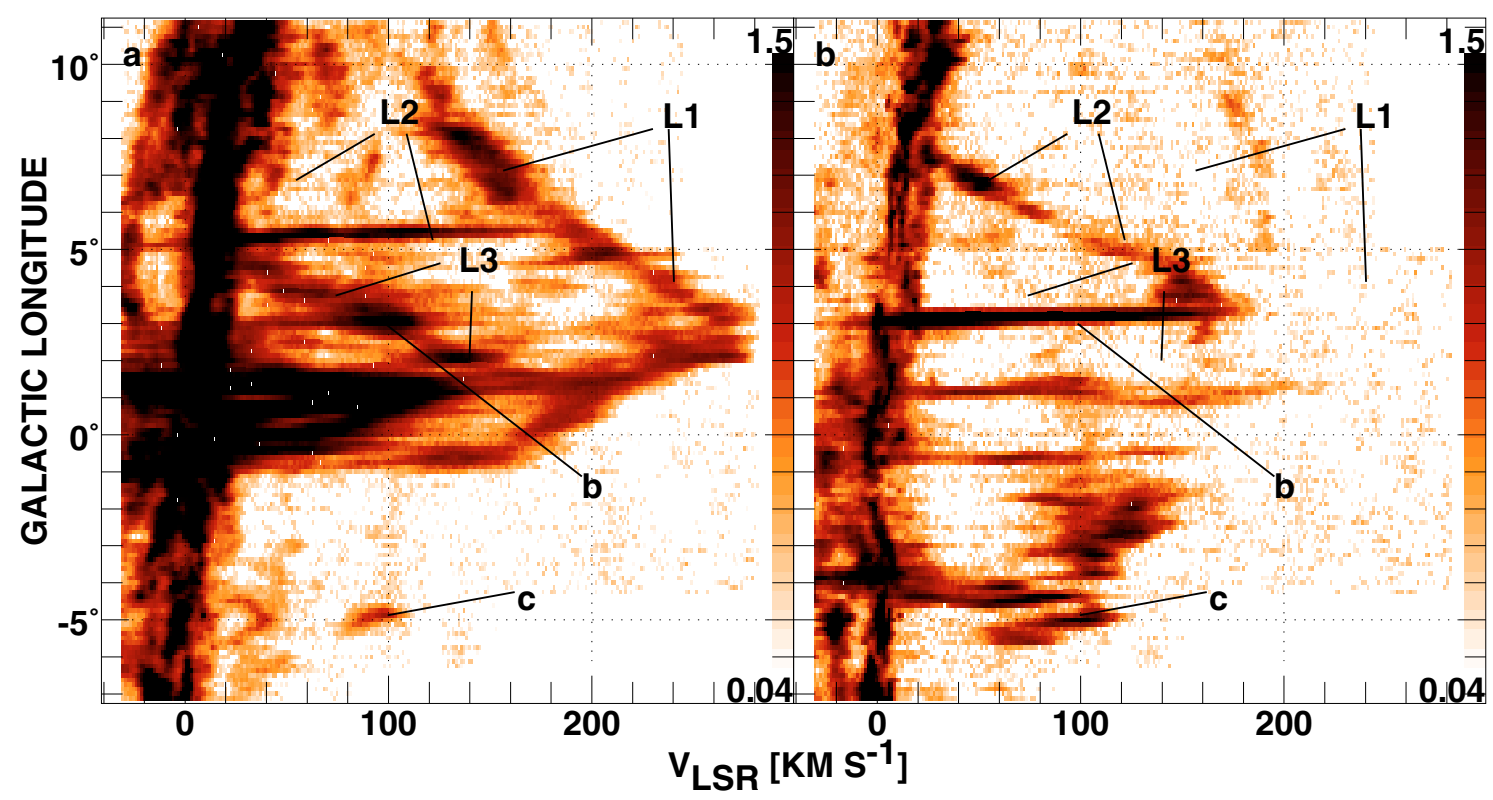

Fig. 2. Positive-velocity portions of $l-v$ diagrams. Left: at negative latitudes averaged over $-1^{\circ} \leq b \leq 0^{\circ}$. Right: at positive latitudes, averaged over $0.5^{\circ} \leq b \leq 1^{\circ}$. The $\mathrm{b}$ and $\mathrm{c}$ notation is repeated from Fig. 1 .

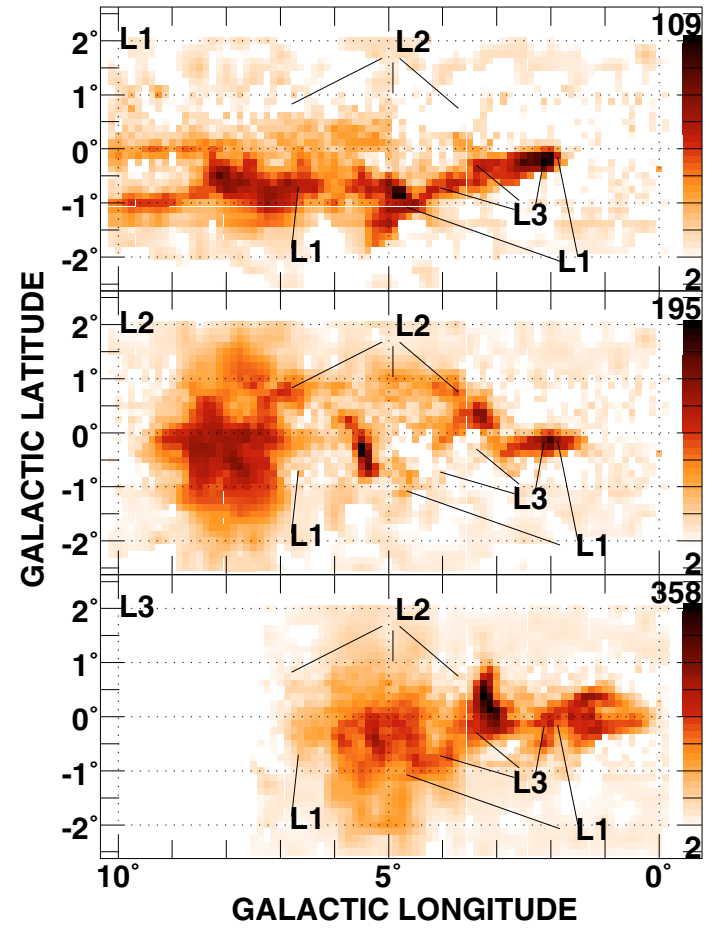

Fig. 3. Maps of $\mathrm{CO}$ brightness in units of $\mathrm{K} \mathrm{km} \mathrm{s}^{-1}$ integrated over the velocities of the L1, L2 and L3 kinematic dust lane features in Fig. 2.

The middle panel integrated over the span of L2 is not particularly revealing of structure in L2 but it inadvertently captures the overall vertical arc of the broad-lined feature at $l=5.5^{\circ}$ from Figs. 1 and 2a, showing that it terminates below at the locus of the L1 dust lane feature (Liszt 2006). Some of the bright vertically-extended emission at $l=8^{\circ}-9^{\circ}$ is low-velocity local gas.

The lowest panel, representing integration over the L3 feature, shows that Clump 2 at $l=3.2^{\circ}$ extends vertically just between L2 and L3. This seems unlikely to be an accident, especially considering the abruptness with which L2 terminates in the vicinity of the broad-lined feature from Clump 2 in Fig. $2 b$. Apparently, L3 underpins the vertically extended Clump 2 at $l=3.2^{\circ}$ in just the same way that $\mathrm{L} 1$ underpins the equivalent vertically-extended broad-lined feature at $l=5.5^{\circ}$.

\section{Vertical structure and secular evolution}

In the bar flow picture, the kinematics are spatially and temporally sequenced as material streams inward from larger radii at higher longitudes. The localized broad-lined features generally represent the uptake and shredding of dense material; the broad features are seen in the emission of $\mathrm{HI}, \mathrm{OH}, \mathrm{CO}, \mathrm{CS}, \mathrm{HCN}$ and the many molecules which require higher density for their excitation, while the large scale dust lanes at $|l|>2^{\circ}$ are seen only in H I and CO (see for instance Fig. 1 of Lee et al. 1999). The multitude of broad-lined features at positive velocity and (mostly) positive longitude is related to the presence of a stronger or richer dust lane on one side of the Galaxy toward the nearer end of the bar.

Presumably there are also a direction and sequence to the vertical structure as well. To interpret the broad-lined feature at $l=5.5^{\circ}$ it was suggested that material is falling down from regions near the equator at the upper extremity of the feature, into the connecting-arm dust lane L1 below the plane (Liszt 2006). However no source of material at the upper extremity was discussed. By contrast, broad-lined Clump 2 at $l=3.2^{\circ}$ has L 2 at its upper extremity and L 3 below, and L 2 does not extend inward of $l=3^{\circ}$. This suggests that the source of the gas being shredded at Clump 2 is still visible in L2, that the sequence is also downward and that it is indeed possible for gas to emerge from a dust lane as well. Why this should occur so far from the galactic equator is a mystery but it should hardly be a surprise if the extremities of Clump 2 and the feature at $l=5.5^{\circ}$ are the site of unusual phenomena.

Understanding the vertical structure of the gas is still a challenge, both observationally and theoretically. Yet, it appears that the secular evolution of the inner galaxy is actually visible in the 
vertical structure and that the tilt of the gas is the result of this evolution.

Acknowledgements. The NRAO is operated by AUI, Inc. under a cooperative agreement with the US National Science Foundation. I thank Tom Dame (CfA) for providing the datacube of Bitran et al. (1997) and thank him and Pat Thaddeus (CfA) for enabling that work. Thanks also to Nemesio Rodriguez-Fernandez and the referee for helpful comments.

\section{References}

Bania, T. M. 1977, ApJ, 216, 381

Bania, T. M. 1980, ApJ, 242, 95

Binney, J., Gerhard, O. E., Stark, A. A., Bally, J., \& Uchida, K. I. 1991, MNRAS, 252, 210

Bitran, M., Alvarez, H., Bronfman, L., May, J., \& Thaddeus, P. 1997, ApJS, 125, 99

Burton, W. B., \& Liszt, H. S. 1978, ApJ, 225, 815

Burton, W. B., \& Liszt, H. S. 1983, A\&AS, 52, 63

Burton, W. B., \& Liszt, H. S. 1993, A\&A, 274, 765
Caldwell, J. A. R., \& Ostriker, J. P. 1981, ApJ, 251, 61

Cohen, R. J., \& Davies, R. D. 1976, MNRAS, 175, 1

Fux, R. 1999, A\&A, 345, 787

Hasegawa, T., Arai, T., Yamaguchi, N., \& Sato, F. 2008, Astrophys. Space. Sci., 313,91

Kaifu, N., Kato, T., \& Iguchi, T. 1972, Nature, 238, 105

Lee, C. W., Lee, H. M., Ann, H. B., \& Kwon, K. H. 1999, ApJ, 513, 242

Liszt, H. S. 2006, A\&A, 447, 533

Liszt, H. S., \& Burton, W. B. 1980, ApJ, 236, 779

Marshall, D. J., Fux, R., Robin, A. C., \& Reylé, C. 2008, A\&A, 477, L21

Oort, J. H. 1977, ARA\&A, 15, 295

Regan, M. W., \& Teuben, P. 2003, ApJ, 599, 999

Regan, M. W., Sheth, K., \& Vogel, S. N. 1999, ApJ, 526, 97

Rodriguez-Fernandez, N. J., Combes, F., Martin-Pintado, J., Wilson, T. L., \& Apponi, A. 2006, A\&A, 455, 963

Sawada, T., Hasegawa, T., Handa, T., \& Cohen, R. J. 2004, MNRAS, 349, 1167

Stark, A. A., \& Bania, T. M. 1986, ApJ, 306, L17

Tanaka, K., Kamegai, K., Nagai, M., \& Oka T. 2007, Publ. Astron. Soc. Jpn, 59, 323

van der Kruit, P. C. 1970, A\&A, 4, 462 